\title{
Pneumococcal vaccination and chronic respiratory diseases
}

This article was published in the following Dove Press journal:

International Journal of COPD

\author{
Filipe Froes ${ }^{1}$ \\ Nicolas Roche ${ }^{2}$ \\ Francesco Blasi ${ }^{3,4}$ \\ 'Chest Department, Hospital Pulido \\ Valente, North Lisbon Hospital \\ Center, Lisbon, Portugal; ${ }^{2}$ Department \\ of Respiratory and Intensive Care \\ Medicine, Cochin Hospital, Paris \\ Descartes University, Paris, France; \\ ${ }^{3}$ Department of Pathophysiology and \\ Transplantation, University of Milan, \\ ${ }^{4}$ Internal Medicine Department, \\ Respiratory Unit and Adult Cystic \\ Fibrosis Center, Fondazione IRCCS ca \\ Granda Ospedale Maggiore Policlinico, \\ Milan, Italy
}

\begin{abstract}
Patients with COPD and other chronic respiratory diseases are especially vulnerable to viral and bacterial pulmonary infections, which are major causes of exacerbations, hospitalization, disease progression, and mortality in COPD patients. Effective vaccines could reduce the burden of respiratory infections and acute exacerbations in COPD patients, but what is the evidence for this? This article reviews and discusses the existing evidence for pneumococcal vaccination efficacy and its changing role in patients with chronic respiratory diseases, especially COPD. Specifically, the recent Community-Acquired Pneumonia Immunization Trial in Adults (CAPITA) showed the efficacy of pneumococcal conjugate vaccine in older adults, many of whom had additional risk factors for pneumococcal disease, including chronic lung diseases. Taken together, the evidence suggests that pneumococcal and influenza vaccinations can prevent community-acquired pneumonia and acute exacerbations in COPD patients, while pneumococcal vaccination early in the course of COPD could help maintain stable health status. Despite the need to prevent pulmonary infections in patients with chronic respiratory diseases and evidence for the efficacy of pneumococcal conjugate vaccine, pneumococcal vaccine coverage and awareness are low and need to be improved. Respiratory physicians need to communicate the benefits of vaccination more effectively to their patients who suffer from chronic respiratory diseases.
\end{abstract}

Keywords: COPD, COPD exacerbation, infection, pneumococcal vaccine

\section{Introduction}

Patients with chronic respiratory diseases (CRDs), especially those with COPD, exhibit altered lung defenses and frequently have multiple comorbidities, which include cardiovascular disease, diabetes, metabolic syndrome, chronic anemia, osteoporosis, depression, and cancer. ${ }^{1-3}$ Such adverse conditions make these patients especially vulnerable to viral and bacterial pulmonary infections, which are major causes of exacerbations, hospitalization, and disease progression. ${ }^{1,4,5}$ Pneumonic infections in COPD patients have a major impact on morbidity, mortality, and health-care costs. ${ }^{6,7}$ Since vaccines are among the most effective means of preventing infectious diseases and their consequences, preventing pulmonary infections in COPD patients through vaccination could reduce the burden of COPD and its complications, as well as further declines in patient health. For many years, pneumococcal vaccination in adults has relied primarily on polysaccharide vaccines. More recently, however, a large study demonstrated the efficacy of pneumococcal conjugate vaccine (PCV) in older adults (Community-Acquired Pneumonia Immunization Trial in Adults [CAPITA]), in which many of the participants had additional risk factors for pneumococcal disease. ${ }^{8}$ Following these new developments, a symposium was held at the 2016 European Respiratory Society International Congress (September 5, 2016, London, UK) to examine and discuss the changing role of pneumococcal vaccination in patients with CRD, especially COPD. One major aim of these discussions was to document whether vaccination
Correspondence: Francesco Blasi Department of Pathophysiology and Transplantation, University of Milan, $7 \mathrm{Via}$ Festa del Perdono, Milan 20I22, Italy Tel +390250320627

Fax +390250320625

Email francesco.blasi@unimi.it (c) (1) (-) 2017 Froes et al. This work is published and licensed by Dove Medical Press Limited. The full terms of this license are available at https://www.dovepress.com/terms.php cc) hereby accept the Terms. Non-commercial uses of the work are permitted without any further permission from Dove Medical Press Limited, provided the work is properly attributed. For permission for commercial use of this work, please see paragraphs 4.2 and 5 of our Terms (https://www.dovepress.com/terms.php). 
with PCV should be included among other strategies known to benefit COPD patients, such as smoking cessation, respiratory therapy, trigger avoidance, and influenza vaccination, to reduce the risk of exacerbations and respiratory events.

\section{Pneumococcal diseases in patients with chronic respiratory diseases}

The global burdens of respiratory diseases remain among the highest of all diseases and the most frequent causes of death. ${ }^{9,10}$ COPD and lower respiratory tract infections (LRTIs) are the third- and fourth-leading causes of death, respectively, after heart disease and stroke, ${ }^{9,11}$ with $\sim 55 \%$ of all LRTI deaths attributable to pneumococcal pneumonia. ${ }^{10}$ In Europe, pneumonia is the leading cause of respiratory hospitalizations and the second leading cause of death due to respiratory disease after lung cancer. ${ }^{12}$ The situation is compounded by the nearly 23 million Europeans with moderate-severe COPD who account for 1.1 million hospitalizations and 150,000 deaths each year. ${ }^{12}$ COPD is frequently associated with comorbidities that contribute greatly to patient mortality. Among these, coronary artery disease and congestive heart failure play a major role. ${ }^{2}$ Hospitalizations and deaths due to COPD can be expected to rise in the future as the disease progresses in the 17 million additional persons in the EU, who are estimated to be living with mild-stage COPD ${ }^{12}$ Currently, pneumonia, COPD, and asthma account for $55.7 \%$ of respiratory hospital admissions and $47.3 \%$ of respiratory deaths in the EU. ${ }^{12}$

Patients with COPD, other CRDs, or other underlying chronic conditions are at high risk of community-acquired pneumonia (CAP), which imposes a substantial disease burden on the European population. Each year, there are an estimated 3.37 million cases of CAP, almost a third of which require hospitalization. ${ }^{12}$ A retrospective cohort analysis of healthcare claims in the US determined the risk of pneumococcal pneumonia to be 2.7 times higher in adults $\geq 65$ years old compared to adults 50-64 years old (Figure 1). ${ }^{13}$ In general, the incidence of pneumococcal pneumonia increased after the age of 65 years and was severalfold higher in persons of all ages living with chronic diseases, such as diabetes, heart disease, asthma, or lung disease, or with underlying conditions, such as smoking or alcoholism, compared to those with no known risk conditions. Among adults $\geq 65$ years old, the incidence of pneumococcal pneumonia was 7.7-fold higher in persons with CRD compared to those without comorbidity (Figure 1). Further, incidence increased exponentially in patients who had multiple comorbidities ("risk stacking"). In patients with three at-risk conditions, incidence was 9-16 times higher than in healthy adults without any known risk factors and twice as high as in immunocompromised patients, who are considered to be at high risk. ${ }^{13}$

The prevalence of chronic conditions that put people at risk is also high, as shown in another US study in which $32.1 \%$ of adults $45-64$ years old and $61.6 \%$ of adults $\geq 65$ years of age had at least two chronic conditions and were considered at high risk of CAP. ${ }^{14}$ As the mean age of the population continues to increase, the prevalence of persons with multiple chronic conditions is also expected to rise. ${ }^{14}$ In Europe, the incidence of CAP was found to be more than 20-fold higher in persons with COPD (22.4 per 1,000 person-years) than in the general population (1.07-1.2 per 1,000 person-years),

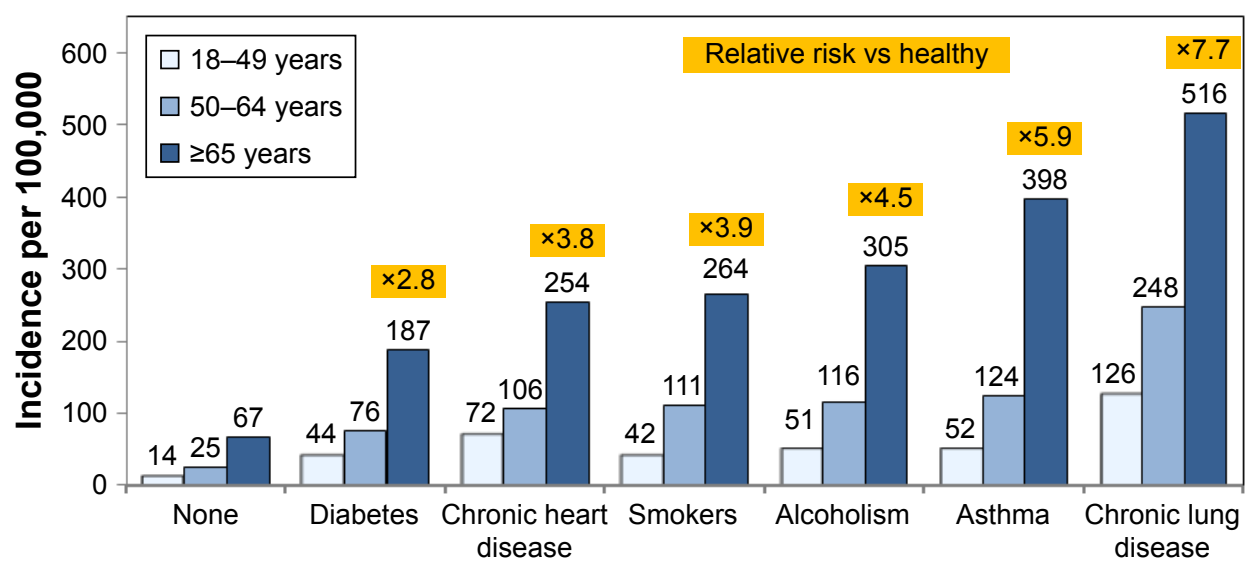

Underlying condition

Figure I Incidence of pneumococcal pneumonia in adults with underlying conditions in the US, 2007-2010.

Notes: By age group and chronic condition. The retrospective cohort analysis utilized health-care claims data from 2007 through 2010 to compare the rates of pneumococcal disease among persons with certain chronic conditions versus their age-matched healthy counterparts. The databases included medical claims (ie, facility and professional services) and outpatient pharmacy claims from private US health plans. Data from Shea et al. ${ }^{13}$ 
with the incidence dramatically higher in persons with severe $\mathrm{COPD}$ (forced expiratory volume in 1 second $\left[\mathrm{FEV}_{1}\right]<50 \%$ of expected). ${ }^{15,16}$ In Sweden, the risk of severe/invasive pneumococcal disease (IPD) was fivefold higher in persons with COPD or pulmonary fibrosis and twice as high in persons with asthma than in the general population. ${ }^{17}$

COPD patients may be more susceptible to respiratory infections, because their mucociliary clearance mechanisms are impaired, and production of the specific cell adhesion molecules that mediate attachment of bacteria and viruses is increased in their airways. Expression of platelet adhesion factor receptor (a cell adhesion molecule for Streptococcus pneumoniae and untypable Haemophilus influenza) and more recently ICAM1 (a cell adhesion molecule for rhinovirus) have been found to be significantly elevated in COPD patients and smokers compared to normal controls. ${ }^{18,19}$ Elevated levels of these proteins may increase the risk of respiratory infection and bacterial colonization. In addition, certain treatments, such as inhaled corticosteroids, can further increase the risk of pneumonia in COPD patients. This risk appears to increase with the dose of the drug, regardless of disease severity, although it does not appear to be associated with a concomitant increase in mortality. ${ }^{20}$

Preventing exacerbations in COPD patients is a major treatment objective. Among the predominant triggers of exacerbations are LRTI, environmental contaminants, pollution, and poor compliance with pulmonary rehabilitation regimes or with long-term oxygen therapy. ${ }^{21}$ However, approximately two-thirds of exacerbations are associated with bacterial or viral LRTI or bacterial colonization of the lungs, ${ }^{1,5,22,23}$ and the spectrum of microorganisms associated with these infections appears to vary with disease severity. ${ }^{24}$ In a study of hospitalized COPD patients, Streptococcus spp. were more prevalent in patients with less severe airway obstruction, whereas Haemophilus spp. were more prevalent in moderate disease and Enterobacteriaceae and Pseudomonas spp. in severe disease. ${ }^{24}$ This finding suggests that pneumococcal vaccination should be envisaged particularly in patients with mild airflow obstruction.

Although there has been some debate on whether pneumonia should be considered a cause or differential diagnosis of acute exacerbation of COPD (AECOPD), another important observation is that pneumonic AECOPD tends to be more severe than nonpneumonic AECOPD. In analyses of COPDrelated hospitalizations, pneumonic AECOPD in Denmark accounted for $36.1 \%$ of all first-time hospitalizations for AECOPD and resulted in more ICU admissions (12.5\% vs
7.7\%), longer median hospitalization stay (9 vs 5 days), and higher 30 -day mortality ( $12 \%$ vs $8 \%$ ) than nonpneumonic AECOPD (Figure 2A-C) ${ }^{25,26}$ The severity of pneumonic AECOPD is further substantiated by a UK study that found in-hospital mortality and 90-day mortality to be significantly higher in patients with pneumonic AECOPD compared to patients with nonpneumonic AECOPD (Figure 2D) ${ }^{27}$ and by a Serbian study that found pneumonia to be the second-most frequent autopsy-confirmed cause of death (28\%) after heart failure (37\%) in COPD patients who died within 24 hours of hospital admission. ${ }^{28}$

Pneumonia not only bodes poorly for the outcome of COPD patients but also has profound implications for healthcare systems. Direct medical costs and productivity costs associated with hospitalization for CAP are twice as high in patients with COPD compared to those in patients with no comorbidity. ${ }^{29}$ COPD patients with CAP are also ninefold more likely to be hospitalized and fourfold more likely to visit the ER, both of which drive the first-year health-care costs for these patients to an excess of US $\$ 22,348$ over the costs of COPD patients without CAP ${ }^{6}$ Excess costs in COPD patients with CAP also remain high over the second year after hospitalization and have been estimated to be $\$ 6,000$. $^{7}$

In summary, the global burden of COPD is substantial, and the risk and frequency of pneumococcal pneumonia in these patients, as well as in others with multiple comorbidities, is many times higher than in healthy adults without comorbidities. Viral and bacterial pneumonic infections are a major cause of respiratory events in COPD patients. The outcomes of these infections tend to be more severe and more deadly than in COPD patients with nonpneumonic AECOPD, leading to longer, more expensive hospitalizations and greater overall costs. Preventing pneumonia in COPD patients could provide substantial benefits to these patients, as well as to the community.

\section{Pneumococcal vaccines: clinical evidence of efficacy and recommendations}

Multivalent pneumococcal polysaccharide vaccines (PPVs) have existed for many years, and the 23 -valent vaccine (PPV23) is recommended by many public health agencies for adults $\geq 65$ years of age and other high-risk groups. Its use comes with possible caveats, however, because the immunoresponses to PPV23 tend to wane over time, and demonstrations of clinical efficacy against CAP and IPD in at-risk populations have been inconsistent. ${ }^{30-32}$ 

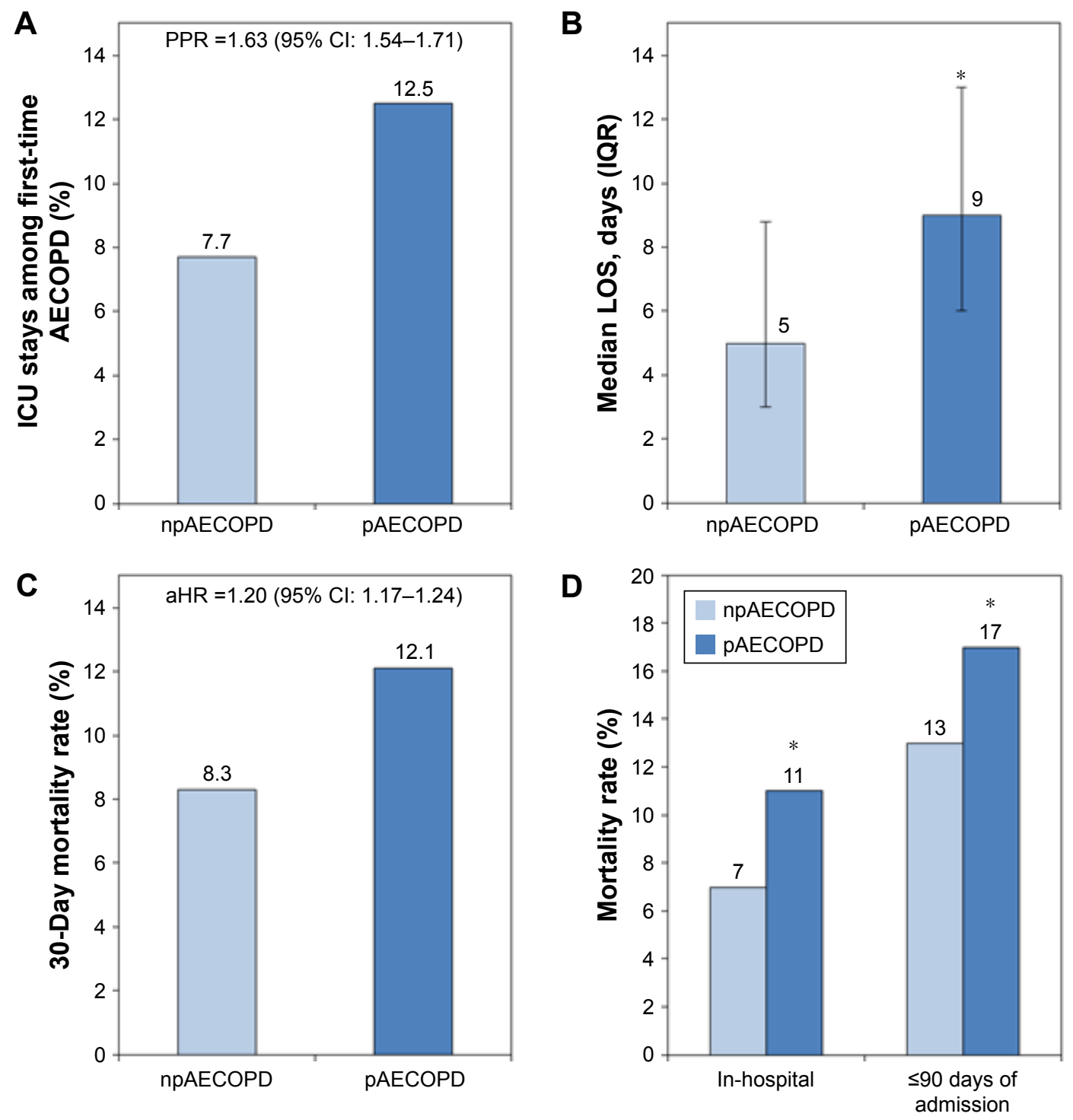

Figure 2 Intensive care unit (ICU) admissions, stays, and mortality of COPD patients with pneumonic and nonpneumonic exacerbations.

Notes: (A) Admission rates among 52,520 patients with a first-time hospitalization for acute COPD exacerbation (AECOPD), $\geq 40$ years of age in Denmark, $2006-2012$. The Danish National Patient Registry was used to identify all first-time inpatient hospitalizations for pneumonic AECOPD (pAECOPD; 8,968 patients) and nonpneumonic AECOPD (npAECOPD; 33,552 patients). All COPD hospitalizations were categorized according to whether they included a primary or secondary diagnosis of pneumonia. Data from Søgaard et al. ${ }^{26}$ (B) Median ICU length of stay (LOS) in COPD patients due to PAECOPD or npAECOPD. Data were obtained from a retrospective analysis of all hospitalizations in 2005 in the departments of internal and respiratory medicine in one Swedish and two Norwegian hospitals. A total of I,I44 hospital admissions (73I patients) were identified from the patient administrative systems: 237 were for PAECOPD (mean age 75.3 years) and 472 for npAECOPD (mean age 71.7 years). $* P<0.00$ I. Data from Andreassen et al. ${ }^{25}$ (C) 30-Day mortality among the 52,520 patients described in A, with adjustments for age, sex, comorbidity, and respiratory medications. Data from Søgaard et al. ${ }^{26}$ (D) In-hospital and 90-day mortality in COPD patients following hospitalization for npAECOPD or pAECOPD in the UK in 2008. UK National COPD audit data were used to determine in-hospital and 90 -day mortality rates of patients admitted with nPAECOPD (542 of 7,833 patients and 95 I of 7,504 patients, respectively) or PAECOPD ( 160 of I,505 patients and 247 of I,44I patients, respectively). A total of 9,338 admissions were assessed, with a mean age of 73 years. $* P<0.00$ I. Data from Myint et al. ${ }^{27}$

However, in a recently updated Cochrane meta-analysis published after our symposium, pneumococcal vaccination (primarily with PPV23) was associated with statistically significant efficacy against CAP and AECOPD in COPD patients. ${ }^{33}$ In contrast, another recent review found the evidence for the clinical efficacy of PPV23 in high-risk and at-risk populations to be inconsistent, despite some short-term protection. ${ }^{34}$ In view of the historically uncertain and variable clinical efficacy of PPV23, the World Health Organization and public health agencies in Europe and the US recognize that there remains an unmet medical need to protect older adults and high-risk groups against pneumococcal pneumonia. ${ }^{35-38}$

Overall, PCVs tend to be more consistently immunogenic than PPVs and to increase the duration and memory of antipneumococcal immunoresponses. Vaccination with 
PPV produces an immunoresponse that is limited to B-cell stimulation and antibody production. ${ }^{39,40}$ In contrast, PCVs contain pneumococcal polysaccharide antigens covalently linked to an immunogenic carrier protein that together induce T-cell-dependent humoral immunoresponses (Figure 3) and stimulate $\mathrm{T}$ cells to help B cells produce antibodies to the vaccine and generate immune memory. ${ }^{40-43}$ These characteristics can improve the magnitude and duration of the initial immunoresponse and potentially allow the immune system to respond more effectively to subsequent exposure to vaccine-type (VT) pneumococcal strains. In studies that have compared humoral immunoresponses to PCV and PPV in normal vaccine-naïve adults or older adults, short-term immunoglobulin and functional antibody responses were generally superior with PCV. ${ }^{40,44-46}$ This has also been demonstrated in COPD patients, in whom superior functional antibody responses persisted for at least 2 years. ${ }^{47,48}$ In some studies, however, the in vitro B-cell-specific responses of older adults vaccinated with PCV or PPV23 did not differ significantly immediately after vaccination ${ }^{49,50}$ or after 6 months. ${ }^{45}$ In healthy adults, long-term immunoresponses with PPV23 remain above baseline for as long as 10 years, though booster immunizations are generally recommended after 5 years. ${ }^{51}$ To our knowledge, the long-term immunogenicity of PCV in normal healthy adults beyond 2 years has not been reported, but significant immunoresponses are known to persist for up to 5 years in HIV-positive adults. ${ }^{52}$

PCVs are recommended for all children and have been shown to prevent nasopharyngeal carriage of VT strains and to protect children against mucosal diseases, IPD, and otitis media. ${ }^{53-57}$ Childhood vaccination programs that include PCV have also demonstrated significant reductions in pneumococcal diseases in adults (herd protection) as an indirect consequence of lower pneumococcal carriage in children. ${ }^{58,59}$

CAPITA was conducted to assess the clinical efficacy of PCV13 in older adults. It was a large prospective randomized, placebo-controlled trial in $>84,000$ participants $\geq 65$ years of age. ${ }^{8}$ The primary outcome was the efficacy of preventing a first episode of VT CAP. With up to 5 years of follow-up, vaccine efficacy in the per-protocol population was $45.6 \%$ against VT CAP and 45\% against abacteremic, noninvasive VT CAP (Figure 4). Vaccine efficacy was 30.6\% against pneumococcal CAP and $75 \%$ against VT IPD. In an exploratory post hoc analysis of the at-risk population in the study, which included over $42 \%$ of the participants in each group, $10 \%$ of which had lung disease, vaccine efficacy was similar: 40.3\% against VT CAP and 30.2\% against pneumococcal CAP.${ }^{97}$ Furthermore, the magnitude and duration of humoral immunoresponses in at-risk adults assessed by opsonophagocytic assay geometric mean antibody titers were

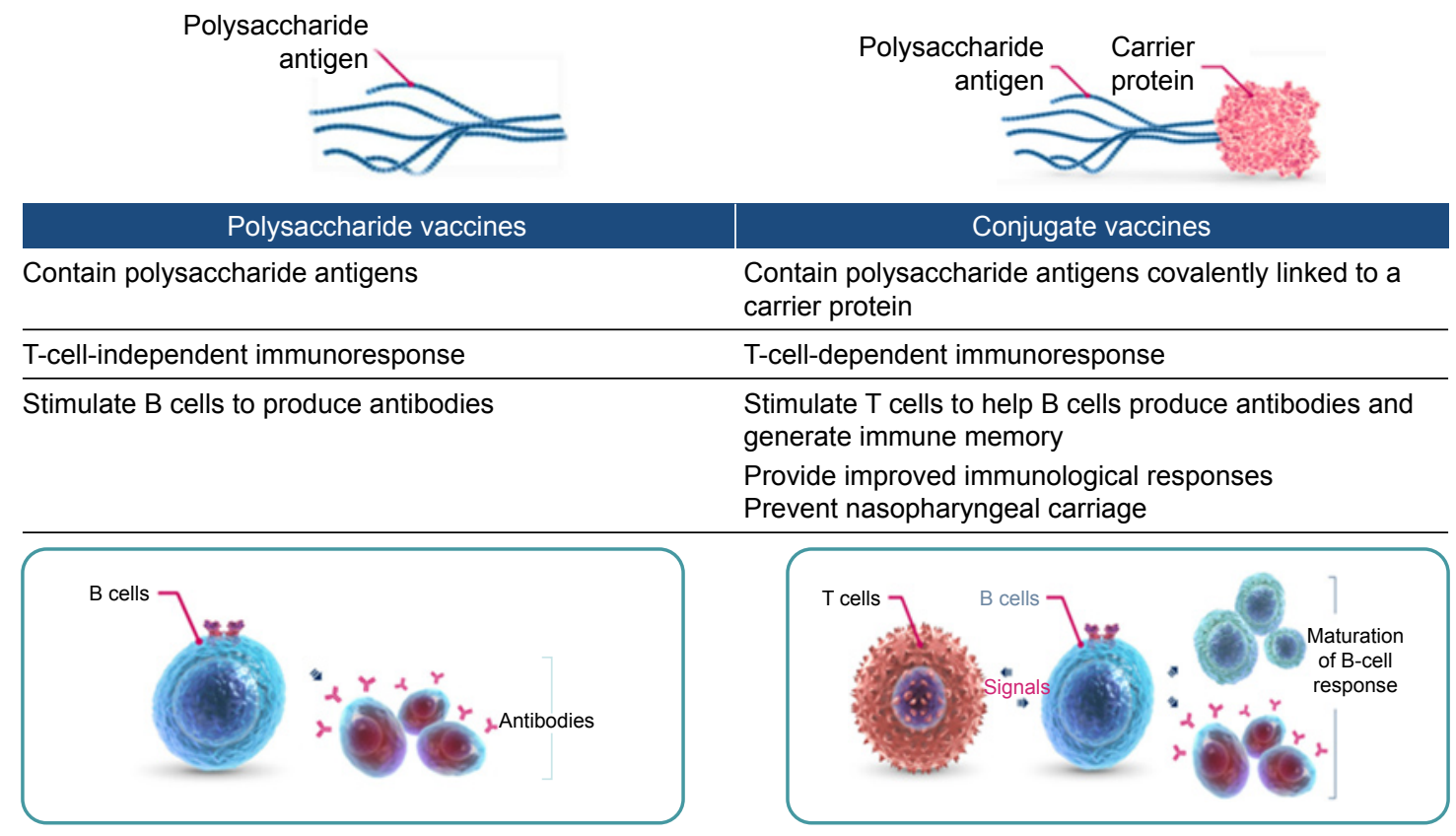

Figure 3 Immunogenic effects of pneumococcal vaccines.

Notes: Pneumococcal polysaccharide vaccines contain serotype-specific polysaccharide antigens only and induce T-cell-independent antibody production from B cells. These responses tend to wane over time. Pneumococcal conjugate vaccines contain serotype-specific polysaccharide antigens covalently linked to a carrier protein. These vaccines induce T-cell-dependent antibody production from B cells and improve immune memory, allowing antipneumococcal immunoresponses to be generated long after vaccination. Based on data from Clutterbuck et al, ${ }^{41}$ de Roux et al, ${ }^{40}$ Pollard et al, ${ }^{42}$ and Siegrist. ${ }^{43}$ 

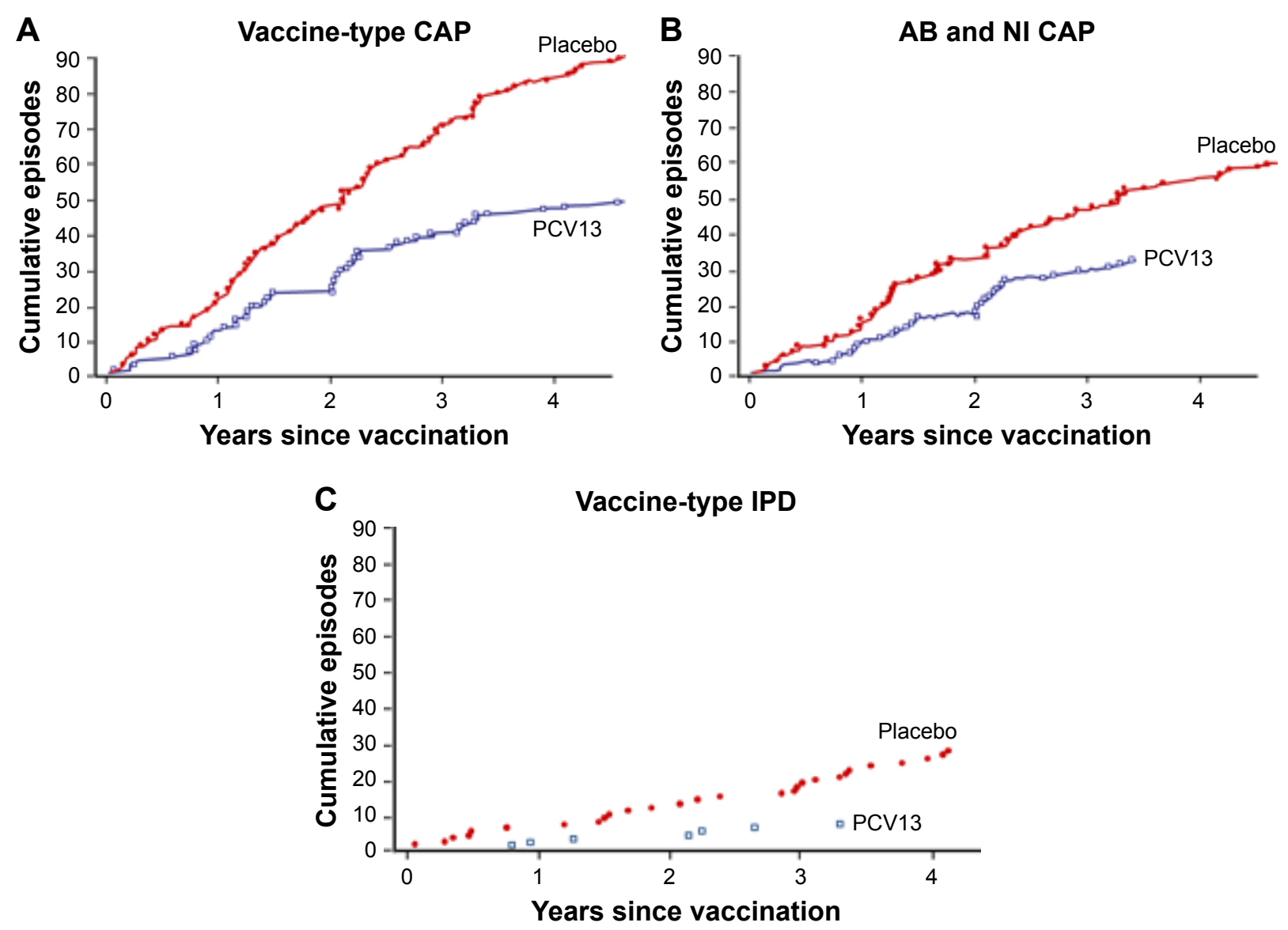

Figure 4 Efficacy of PCVI3 in older adults: results of the CAPITA study.

Notes: CAPITA was a randomized, double-blind, placebo-controlled trial that evaluated the efficacy of I3-valent polysaccharide conjugate vaccine (PCVI3) versus placebo in 84,496 adults $\geq 65$ years of age. The primary objective was vaccine efficacy in preventing a first episode of vaccine-type pneumococcal community-acquired pneumonia (CAP). A secondary objective was vaccine efficacy in preventing a first episode of vaccine-type abacteremic $(A B)$ and noninvasive $(\mathrm{NI})$ pneumococcal CAP. The figure illustrates post hoc analyses of the cumulative episodes of primary and secondary efficacy end points in the per-protocol population. (A) Cumulative first episodes of vaccine-type CAP from vaccination to 5 -year follow-up. Vaccine efficacy was $45.6 \%$ (95.2\% Cl: $21.8 \%-62.5 \%, P<0.001)$. (B) Cumulative first episodes of vaccine-type AB and NI CAP from vaccination to 5 -year follow-up. Vaccine efficacy was $45 \%$ (95.2\% Cl: $14.2 \%-65.3 \%, P=0.007)$. (C) Cumulative first episodes of vaccine-type invasive pneumococcal disease (IPD) from vaccination to 5-year follow-up. Vaccine efficacy was $75 \%$ ( $95 \% \mathrm{Cl}: 41.4 \%-90.8 \%, P<0.00 \mathrm{I})$. From $\mathrm{N}$ Engl J Med. Bonten et al. Polysaccharide conjugate vaccine against pneumococcal pneumonia in adults. 372:I I 14-I I25. Copyright @ 2015 Massachusetts Medical Society. Reprinted with permission from Massachusetts Medical Society. ${ }^{8}$

Abbreviation: CAPITA, Community-Acquired Pneumonia Immunization Trial in Adults.

comparable to those of the overall population and persisted for at least 2 years. ${ }^{60}$

Finally, another post hoc analysis predicted that vaccine efficacy would decline from $65 \%$ for adults 65 years of age to $40 \%$ for adults 75 years of age, suggesting that patients with indications of PCV should be vaccinated as early as possible. ${ }^{61}$ While these results are encouraging, post hoc analyses should be viewed with caution, and the efficacy of PCV13 for preventing VT CAP in CRD patients has yet to be demonstrated in a randomized clinical trial designed to address this issue specifically. It should be noted that in such a hypothetical trial, the necessary sample size to demonstrate efficacy is likely to be very large, and recruiting the minimum number of CRD patients for the treatment and control arms would be extremely difficult, especially in the context of populations benefiting from herd immunity arising from pediatric vaccination programs.
Recently published studies have also investigated the efficacy of PPV23 in older adults. A meta-analysis of 17 eligible studies in adults $\geq 60$ years of age found pooled efficacies of PPV23 against IPD (45\%-73\%) and pneumococcal pneumonia $(48 \%-64 \%)$ by any serotype. ${ }^{62}$ Another meta-analysis of studies in adults $\geq 50$ years of age also found evidence of significant vaccine efficacy for PPV23 against IPD (50\%-54\%) and against all-cause CAP $(4 \%-17 \%){ }^{63}$ Both studies concluded that efficacies of PPV23 and PCV13 against VT pneumococcal disease were comparable. In a multicenter prospective study in adults $\geq 65$ years of age treated for CAP, PPV23 efficacy was $27.4 \%$ (95\% CI: $3.2 \%-45.6 \%$ ) against all pneumococcal pneumonia. ${ }^{64}$

The US Advisory Committee on Immunization Practices currently recommends PCV13 followed by PPV23 for all adults $\geq 65$ years of age who have not previously received pneumococcal vaccine and in persons $\geq 19$ years of age who 
are at high risk of pneumococcal disease due to underlying medical conditions. ${ }^{65,66}$ Several European scientific societies also recommend PCV13 for immunocompromised adults and all persons $\geq 65$ years of age, along with at least one dose of PPV23 given 2-6 months afterward. ${ }^{67}$ PCV13 is now being progressively implemented into national programs across Europe. As of August 2016, PCV13 is recommended for use in high-risk populations in $>25$ countries, for use in at-risk populations in $>16$ countries, and for use in older adults ( $>50$ or $>65$ years of age) in $>14$ countries.

PCV13 is recommended to be given before PPV23. ${ }^{66}$ This schedule is based on the observation that the responses to the serotypes common to both vaccines were stronger if PCV13 were administered first. ${ }^{68}$ Of note, PCV13 can be given concomitantly with a seasonal inactivated influenza vaccine without impacting immunoresponses to either vaccine, which can help avoid missed opportunities for vaccination. ${ }^{69,70}$

In summary, PCV13 has been developed to provide improved protection for older adults and adults with chronic conditions, such as COPD, who are at high risk of pneumococcal infection and pneumonia. In the pivotal CAPITA study, PCV13 induced specific and efficacious immunoresponses in adults $\geq 65$ years of age with or without underlying chronic conditions that elevate the risk of pneumonia, and vaccine efficacy against VT CAP and VT IPD persisted for up to 5 years. As a result, an increasing number of countries across Europe are including PCV13 in their vaccination recommendations.

\section{Role of pneumococcal vaccination in patients with chronic respiratory diseases}

Current European Respiratory Society and European Society of Clinical Microbiology and Infectious Diseases guidelines were published before the recent results on PCV, and thus recommended only PPV23 for older adults and adults with risk factors for pneumococcal disease, such as age $\geq 65$ years, congestive heart failure, COPD, and a previous history of pneumonia. ${ }^{71}$ Specific recommendations on pneumococcal vaccination are lacking in many guidelines on CRDs from international societies. For COPD, pneumococcal vaccination is recommended in current GOLD guidelines, which mention the increased immunogenicity of PCV13, but do not propose a clear position on whether it should be preferred over PPV23. ${ }^{72}$

Because LRTIs are an important trigger for exacerbations, preventing them in COPD patients could also help prevent exacerbations. ${ }^{5}$ Early prevention of exacerbations may also be critically important for preventing disease progression and subsequent events, as exacerbations can indicate or precede a feed-forward pattern of increased susceptibility to exacerbation and in some cases a frequent exacerbation phenotype. ${ }^{21}$ In the natural history of COPD, each exacerbation is associated with an increased risk of subsequent exacerbations, which tend to occur with greater frequency and severity. It is well known that patients can enter a vicious cycle of infection, exacerbation, and disease progression. ${ }^{3,4,73,74} \mathrm{With}$ each exacerbation, further tissue destruction and functional impairment can occur along with worsening mucociliary clearance and increased bacterial colonization, all of which increase the risk of developing an LRTI and another exacerbation. The reality of this concept was further demonstrated by comparing the exacerbation frequencies of COPD patients after a CAP episode to those in COPD patients who had not had CAP (Figure 5). The cumulative risk of exacerbation in the first year after CAP was 1.5 times higher in patients who had had CAP (17\%) compared to those who had not $(11.4 \% ; P<0.001) .{ }^{96}$

\section{Clinical evidence of pneumococcal vaccine efficacy in COPD}

In principle, pneumococcal vaccination should prevent LRTI and acute exacerbations in COPD patients, but interpreting the existing evidence for the clinical efficacy of PPV in these high-risk patients has been somewhat problematic. In a 2010 Cochrane systematic review and meta-analysis of seven studies, ${ }^{32}$ the overall vaccine effect against pneumonia was of borderline significance compared to placebo, with an OR of 0.72 (95\% CI: 0.51-1.01), with no significant effects on either exacerbations or cardiorespiratory mortality. While these results suggested that PPV may confer some protection in COPD patients, the lack of statistical significance for any outcome and the heterogeneity of the studies rendered the evidence inconclusive. The evidence for vaccine efficacy improved somewhat in the 2017 update of this Cochrane review, which included a reanalysis of the same studies used in the 2010 meta-analysis of pneumococcal vaccination efficacy (primarily PPV23) against CAP, and concluded that vaccination was associated with significant reductions in the likelihood of experiencing CAP (OR: 0.62, 95\% CI: 0.43-0.89) or AECOPD (OR: 0.6, 95\% CI: 0.39-0.9). ${ }^{33}$

A recent review of pneumococcal vaccine efficacy studies in persons with underlying risk factors, such as COPD or a CRD, vaccine efficacy against any-cause CAP was found to vary widely between $-338 \%$ and $43 \%$, with an overall efficacy of $29 \%(95 \% \mathrm{CI}:-39 \%-63 \%)$ in adults aged $\geq 50$ years with CRD. ${ }^{34}$ In a randomized controlled trial in 596 COPD patients, PPV23 efficacy against CAP was significant at 76\% (95\% CI: 20-93, $P=0.013$ ) in patients $<65$ years of age, nearly significant at 48\% (95\% CI: $27-80, P=0.076)$ in those with severe airflow obstruction $\left(<40 \% \mathrm{FEV}_{1}\right)$, and 


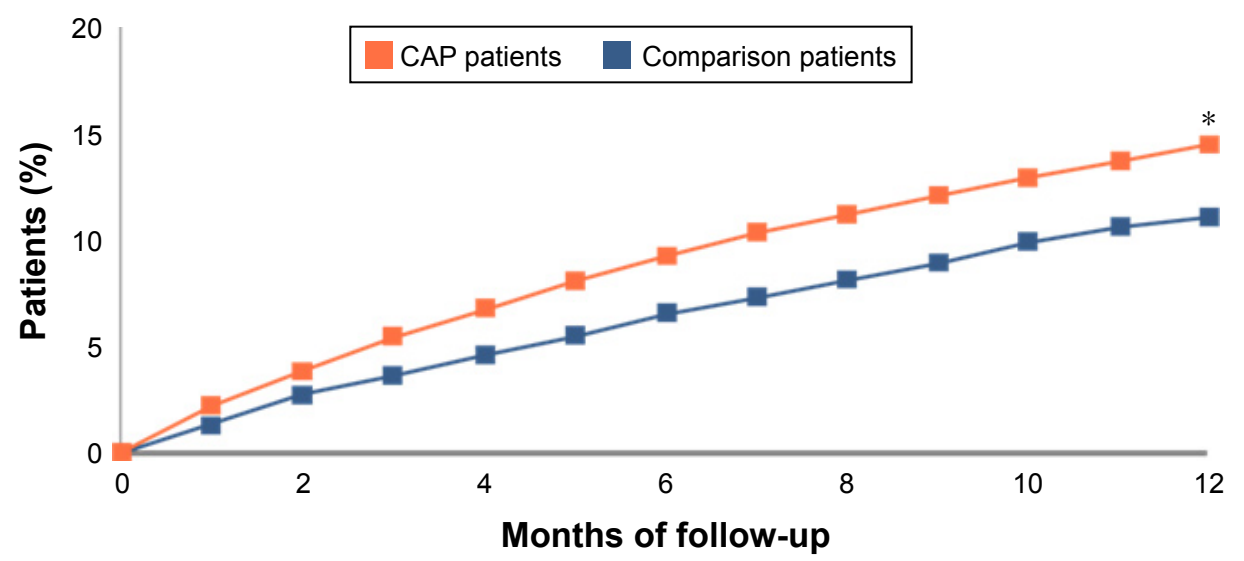

Number of patients at risk

\begin{tabular}{|c|c|c|c|c|c|c|c|}
\hline CAP patients & 8,274 & 7,493 & 6,891 & 6,384 & 5,908 & 5,412 & 4,984 \\
\hline $\begin{array}{l}\text { Comparison } \\
\text { patients }\end{array}$ & 8,274 & 7,715 & 7,273 & 6,847 & 6,372 & 5,889 & 5,460 \\
\hline \multicolumn{8}{|c|}{ Percentage with a subsequent exacerbation } \\
\hline CAP patients & - & $3.9 \%$ & $6.8 \%$ & $9.3 \%$ & $11.2 \%$ & $12.9 \%$ & $14.6 \%$ \\
\hline $\begin{array}{l}\text { Comparison } \\
\text { patients }\end{array}$ & - & $2.8 \%$ & $4.7 \%$ & $6.6 \%$ & $8.1 \%$ & $9.9 \%$ & $11.2 \%$ \\
\hline
\end{tabular}

Figure 5 Community-acquired pneumonia (CAP) and occurrence of subsequent exacerbations in COPD patients in the US, 2010.

Notes: In a retrospective cohort study of 12,327 matched pairs, CAP patients aged $\geq 18$ years who had continuous and comprehensive health benefits, evidence of COPD, and no evidence of pneumonia during the preceding year were identified between January and December 20I0. One comparison patient from the corresponding source population who had continuous and comprehensive health benefits, evidence of COPD, and no evidence of pneumonia during the preceding year was matched with each CAP patient on the date of the initial CAP encounter (or inpatient admission date) or within the next 30 days. Comparison patients were also matched with each CAP patient on age $( \pm I$ year), sex, and selected markers of health status measured in the preceding year. Exacerbation was defined as a hospitalization or emergency department visit during follow-up, which began 30 days after pneumonia diagnosis and ended 12 months later. ${ }^{*} P<0.001$. From Weycker et al; ${ }^{96}$ reprinted with permission from the authors.

strongest for both factors combined (91\% [95\% CI: 35-99, $P=0.002]) .{ }^{75}$ However, vaccine efficacy was not significant for the overall cohort (24\% [95\% CI: $-24-54, P=0.333])$.

In a study of the combined efficacy of PPV23 and influenza vaccination in preventing acute exacerbation in adult CRD patients, acute exacerbation frequency was significantly lower in the PPV23 + influenza vaccination group than in the influenza vaccination-alone group $(P=0.022)$, and there was a significant additive effect of PPV23 in COPD patients $(P=0.037) .{ }^{76}$ In view of the limited numbers of studies available to date, the evidence on the role of PPV for protecting COPD patients from CAP can be considered inconsistent.

In contrast, seasonal influenza vaccine has demonstrated significant efficacy in preventing exacerbations in COPD patients compared to placebo, with a reported weighted mean difference between treatments of -0.37 (95\% CI: -0.64 to $-0.11, P=0.006$ ) in a meta-analysis of two randomized trials. ${ }^{77}$ If influenza vaccine is administered concomitantly with PPV23, the two vaccines provide an additive effect in reducing the risk of hospitalization for pneumonia, death, death from influenza, and death from pneumonia. ${ }^{78-80}$

Another potential benefit of an effective pneumococcal vaccination could be a reduction in antibiotic treatments.
This has not been studied in COPD patients, but has been estimated in children by comparing antibiotic treatment days for $S$. pneumoniae infections in countries with high childhood PCV coverage versus countries without such coverage. ${ }^{81}$ Globally, an estimated 11.4 million antibiotic treatment days were avoided in countries with PCV coverage, a reduction of $47 \%$. Reducing antibiotic treatment in COPD patients could also help delay or prevent colonization of the lungs by antibiotic resistant bacteria.

\section{Real-life vaccine coverage of the target population}

Despite the availability of effective pneumococcal vaccines and recommendations from most national health authorities, coverage remains suboptimal in many settings. Recent coverage rates have been fairly high in the UK (70\% in persons $\geq 65$ years of age in 2014-2015) and in Spain (76\% in persons $\geq 60$ years of age in 2010 ), lower in Ireland (36\% in persons $\geq 65$ years of age and $18 \%$ in highrisk adults in 2013), and very low in Norway (15\%-30\% in persons $\geq 65$ years of age in 2014-2015), Germany (15\% in high-risk persons in 2014$)$, and France ( $5 \%$ in persons $\geq 65$ years of age in 2010-2011). ${ }^{82-87}$ 
An indication of the reasons behind these suboptimal coverage rates may lie in the perceptions and vaccine awareness of older adults. A recent survey (PneuVUE, Pfizer, Paris, France) measured public awareness of pneumonia and vaccine perceptions in adults $\geq 50$ years of age across nine EU countries. ${ }^{88}$ The survey found that $85 \%$ of these adults trusted vaccines, $27 \%$ avoided vaccines (for safety reasons), and $29 \%$ feel they "didn't need" vaccines because they were not at risk. However, high proportions of respondents reported that they did follow their physician's advice (92\%) and that they preferred to obtain additional medical information from their doctor (92\%). Overall, pneumococcal vaccine awareness was low, and the most frequent reason given (55\%) for not being vaccinated was that it was never offered by their doctor.

To summarize, evidence-based guidelines recommend pneumococcal vaccination in persons with CRD, and PCV13 has already been included in a number of national recommendations, including those in the US, France, Portugal, Spain, Italy, and others. ${ }^{66,89-92}$ Some countries, such as Germany and the UK, continue to consider the evidence and favor the broader serotype coverage of PPV23. ${ }^{93,94}$ In patients with COPD, preventing LRTIs, which frequently involve S. pneumoniae, appears critical, because they can alter disease status and speed disease progression, thus increasing the risk of subsequent exacerbation. Including pneumococcal vaccination in early care for COPD may improve the long-term natural history of the disease, although further evidence is needed. Despite recommendations, overall pneumococcal vaccination coverage is poor across Europe and public awareness and knowledge of pneumococcal disease and vaccines is low. Since patients rely on their physicians as the main source of health information, physicians need to do a better job of informing their at-risk patients about the dangers of pneumonia and the benefits of pneumococcal vaccination.

\section{Conclusion}

Global CRD burdens are high and contribute to substantial excess health-care use, hospitalizations, and global morbidity and mortality. ${ }^{72}$ Patients with COPD and other CRDs are at high risk of CAP, IPD, and exacerbations that further increase health-care burdens and mortality. ${ }^{12,13}$ In COPD, airway infections are important triggers of exacerbations and hasten disease progression. There is evidence for the efficacy of PPV23 in older adults, ${ }^{62,63}$ but evidence for protection in CRD patients has been considered inconclusive. ${ }^{32}$ The CAPITA study provided clinical evidence that PCV13 is effective at preventing CAP in older adults, including those with comorbidities. ${ }^{8}$ Post hoc analyses of CAPITA are consistent with PCV13 efficacy in patients with comorbidities, but these results should be viewed with caution. Indeed, vaccine efficacy in adults is likely to vary according to the pneumococcal vaccines used in pediatric vaccination programs and efficacy may be low or difficult to demonstrate in settings where pediatric uptake of pneumococcal vaccine is high and the consequent burden of disease in adults is reduced. ${ }^{95}$ However, given the substantially higher risk of respiratory infection and dire consequences of such infections in patients with COPD or CRD, vaccination is warranted in these patients, regardless of the level of herd protection present in the community.

Influenza and pneumococcal infections are important contributors to CAP and acute exacerbations in COPD patients, and pneumococcal and influenza vaccinations together may have an additive preventive effect. ${ }^{78-80}$ Vaccination early in the course of COPD could help maintain stable health status, although further demonstration is needed. ${ }^{5,21}$ Despite the great burden of pneumonia and pneumococcal infection, the need for vaccination in patients with CRD-COPD, the evidence on pneumococcal vaccine efficacy, and widespread national recommendations, improvements are needed in pneumococcal vaccine coverage, knowledge, and awareness. ${ }^{88}$ As the main source of medical information for the public, physicians need to communicate more effectively the benefits of vaccination with PCV to their patients, especially those with CRDs.

\section{Acknowledgments}

The content of this article is based on a symposium (sponsored by Pfizer Inc., Paris, France) held at the ERS International Congress in London in September 2016. The authors take full responsibility for the content of this article and thank Dr Kurt Liittschwager (4Clinics, Paris, France) for scientific writing services and assistance in preparing the manuscript in close collaboration with the authors. The authors also thank Dr Tin Tin Htar (Pfizer) for valuable comments and suggestions. Financial support for the preparation of this manuscript was provided by Pfizer.

\section{Disclosure}

FF, NR, and FB received personal fees from Pfizer to conduct the symposium. FF has also received grants, personal fees, and nonfinancial support from Pfizer; personal fees and nonfinancial support from MSD; personal fees from Sanofi Pasteur and Novartis; and nonfinancial support from Sanofi, AstraZeneca, Bayer, and Bial. NR has received grants and personal fees from Pfizer, Boehringer Ingelheim, and Novartis, and personal fees from Teva, GSK, AstraZeneca, Chiesi, Mundipharma, Cipla, Sanofi, Sandoz, 3M, and Zambon. 
FB has received grants and personal fees from Bayer, Chiesi, Guidotti, Menarini, Pfizer, Teva, and Zambon, and personal fees from Dompé, AstraZeneca, and Novartis. The authors report no other conflicts of interest in this work.

\section{References}

1. Celli BR, Barnes PJ. Exacerbations of chronic obstructive pulmonary disease. Eur Respir J. 2007;29(6):1224-1238.

2. Divo M, Cote C, de Torres JP, et al. Comorbidities and risk of mortality in patients with chronic obstructive pulmonary disease. Am J Respir Crit Care Med. 2012;186(2):155-161.

3. Sethi S. Infection as a comorbidity of COPD. Eur Respir J. 2010; 35(6):1209-1215.

4. Sethi S, Murphy TF. Infection in the pathogenesis and course of chronic obstructive pulmonary disease. $N$ Engl J Med. 2008;359(22): 2355-2365.

5. Wedzicha JA, Mackay AJ, Singh R. COPD exacerbations: impact and prevention. Breathe. 2013;9(6):434-440.

6. Lin J, Li Y, Tian H, et al. Costs and health care resource utilization among chronic obstructive pulmonary disease patients with newly acquired pneumonia. Clinicoecon Outcomes Res. 2014;6: 349-356.

7. Ryan M, Suaya JA, Chapman JD, Stason WB, Shepard DS, Thomas CP. Incidence and cost of pneumonia in older adults with COPD in the United States. PLoS One. 2013;8(10):e75887.

8. Bonten MJ, Huijts SM, Bolkenbaas M, et al. Polysaccharide conjugate vaccine against pneumococcal pneumonia in adults. NEngl J Med. 2015; 372(12):1114-1125.

9. Vos T, Allen C, Arora M, et al. Global, regional, and national incidence, prevalence, and years lived with disability for 310 diseases and injuries, 1990-2015: a systematic analysis for the Global Burden of Disease Study 2015. Lancet. 2016;388(10053):1545-1602.

10. Wang H, Naghavi M, Allen C, et al. Global, regional, and national life expectancy, all-cause mortality, and cause-specific mortality for 249 causes of death, 1980-2015: a systematic analysis for the Global Burden of Disease Study 2015. Lancet. 2016;388(10053): 1459-1544.

11. World Health Organization. The top 10 causes of death. 2014. Available from: http://www.who.int/mediacentre/factsheets/fs310/en. Accessed December 31, 2016.

12. European Respiratory Society. European Lung White Book. Lausanne, Switzerland: ERS; 2016.

13. Shea KM, Edelsberg J, Weycker D, Farkouh RA, Strutton DR, Pelton SI. Rates of pneumococcal disease in adults with chronic medical conditions. Open Forum Infect Dis. 2014;1(1):ofu024.

14. Ward BW, Black LI. State and regional prevalence of diagnosed multiple chronic conditions among adults aged $\geq 18$ years: United States, 2014. MMWR Morb Mortal Wkly Rep. 2016;65(29):735-738.

15. Mullerova H, Chigbo C, Hagan GW, et al. The natural history of community-acquired pneumonia in COPD patients: a population database analysis. Respir Med. 2012;106(8):1124-1133.

16. Torres A, Peetermans WE, Viegi G, Blasi F. Risk factors for communityacquired pneumonia in adults in Europe: a literature review. Thorax. 2013;68(11):1057-1065.

17. Inghammar M, Engström G, Kahlmeter G, Ljungberg B, Löfdahl CG, Egesten A. Invasive pneumococcal disease in patients with an underlying pulmonary disorder. Clin Microbiol Infect. 2013;19(12): $1148-1154$.

18. Shukla SD, Mahmood MQ, Weston S, et al. The main rhinovirus respiratory tract adhesion site (ICAM-1) is upregulated in smokers and patients with chronic airflow limitation (CAL). Respir Res. 2017;18(1):6.

19. Shukla SD, Muller HK, Latham R, Sohal SS, Walters EH. Plateletactivating factor receptor (PAFr) is upregulated in small airways and alveoli of smokers and COPD patients. Respirology. 2016;21(3): 504-510.
20. Yawn BP, Li Y, Tian H, Zhang J, Arcona S, Kahler KH. Inhaled corticosteroid use in patients with chronic obstructive pulmonary disease and the risk of pneumonia: a retrospective claims data analysis. Int $J$ Chron Obstruct Pulmon Dis. 2013;8:295-304.

21. Wedzicha JA, Brill SE, Allinson JP, Donaldson GC. Mechanisms and impact of the frequent exacerbator phenotype in chronic obstructive pulmonary disease. BMC Med. 2013;11:181.

22. Sapey E, Stockley RA. COPD exacerbations - 2: aetiology. Thorax. 2006;61(3):250-258.

23. Papi A, Bellettato CM, Braccioni F, et al. Infections and airway inflammation in chronic obstructive pulmonary disease severe exacerbations. Am J Respir Crit Care Med. 2006;173(10):1114-1121.

24. Eller J, Ede A, Schaberg T, Niederman MS, Mauch H, Lode H. Infective exacerbations of chronic bronchitis: relation between bacteriologic etiology and lung function. Chest. 1998;113(6): $1542-1548$.

25. Andreassen SL, Liaaen ED, Stenfors N, Henriksen AH. Impact of pneumonia on hospitalizations due to acute exacerbations of COPD. Clin Respir J. 2014;8(1):93-99.

26. Søgaard M, Madsen M, Løkke A, Hilberg O, Sørensen HT, Thomsen RW. Incidence and outcomes of patients hospitalized with COPD exacerbation with and without pneumonia. Int J Chron Obstruct Pulmon Dis. 2016;11:455-465.

27. Myint PK, Lowe D, Stone RA, Buckingham RJ, Roberts CM. U.K. National COPD Resources and Outcomes Project 2008: patients with chronic obstructive pulmonary disease exacerbations who present with radiological pneumonia have worse outcome compared to those with non-pneumonic chronic obstructive pulmonary disease exacerbations. Respiration. 2011;82(4):320-327.

28. Zvezdin B, Milutinov S, Kojicic M, et al. A postmortem analysis of major causes of early death in patients hospitalized with COPD exacerbation. Chest. 2009;136(2):376-380.

29. Polsky D, Bonafede M, Suaya JA. Comorbidities as a driver of the excess costs of community-acquired pneumonia in U.S. commercially-insured working age adults. BMC Health Serv Res. 2012; $12: 379$.

30. Andrews NJ, Waight PA, George RC, Slack MP, Miller E. Impact and effectiveness of 23-valent pneumococcal polysaccharide vaccine against invasive pneumococcal disease in the elderly in England and Wales. Vaccine. 2012;30(48):6802-6808.

31. Artz AS, Ershler WB, Longo DL. Pneumococcal vaccination and revaccination of older adults. Clin Microbiol Rev. 2003;16(2): 308-318.

32. Walters JA, Smith S, Poole P, Granger RH, Wood-Baker R. Injectable vaccines for preventing pneumococcal infection in patients with chronic obstructive pulmonary disease. Cochrane Database Syst Rev. 2010;(11):CD001390.

33. Walters JA, Tang JN, Poole P, Wood-Baker R. Pneumococcal vaccines for preventing pneumonia in chronic obstructive pulmonary disease. Cochrane Database Syst Rev. 2017;1:CD001390.

34. Htar MT, Stuurman AL, Ferreira G, et al. Effectiveness of pneumococcal vaccines in preventing pneumonia in adults, a systematic review and meta-analyses of observational studies. PLoS One. 2017; 12(5):e0177985.

35. [No authors listed]. Pneumococcal vaccines WHO position paper: 2012. Wkly Epidemiol Rec. 2012;87(14):129-144.

36. Khoie T, Tiernan R, deVore N. Vaccines and Related Biological Products Advisory Committee. FDA Briefing Document - Prevnar 13 (PCV13): pneumococcal 13-valent conjugate vaccine (diphtheria CRM197 protein). 2011. Available from: https://pdfs.semanticscholar. org/d394/d5e91b28e01e28c89a81c312dd79b02007b9.pdf. Accessed October 11, 2017.

37. deVore N. Summary basis for regulatory action [Prevnar 13/ pneumococcal 13-valent conjugate vaccine (diphtheria CRM197 protein)]. 2011. Available from: http://www.fda.gov/downloads/ BiologicsBloodVaccines/Vaccines/ApprovedProducts/UCM287412. pdf. Accessed October 1, 2017. 
38. European Medicines Agency. CHMP variation assessment report [Prevenar 13]: type II variation EMEA/H/C/001104/II/0028. 2011. Available from: http://www.ema.europa.eu/docs/en_GB/document library/EPAR_-_Assessment_Report_-_Variation/human/001104/ WC500119784.pdf. Accessed October 1, 2017.

39. Clutterbuck EA, Lazarus R, Yu LM, et al. Pneumococcal conjugate and plain polysaccharide vaccines have divergent effects on antigen-specific B cells. J Infect Dis. 2012;205(9):1408-1416.

40. de Roux A, Schmöle-Thoma B, Siber GR, et al. Comparison of pneumococcal conjugate polysaccharide and free polysaccharide vaccines in elderly adults: conjugate vaccine elicits improved antibacterial immune responses and immunological memory. Clin Infect Dis. 2008; 46(7):1015-1023.

41. Clutterbuck EA, Salt P, Oh S, Marchant A, Beverley P, Pollard AJ. The kinetics and phenotype of the human B-cell response following immunization with a heptavalent pneumococcal-CRM conjugate vaccine. Immunology. 2006;119(3):328-337.

42. Pollard AJ, Perrett KP, Beverley PC. Maintaining protection against invasive bacteria with protein-polysaccharide conjugate vaccines Nat Rev Immunol. 2009;9(3):213-220.

43. Siegrist CA. Vaccine immunology. In: Plotkin SA, Orenstein W, Offitt PA, editors. Vaccines. 5th ed. Amsterdam, the Netherlands: Elsevier; 2008:17-36.

44. Goldblatt D, Southern J, Andrews N, et al. The immunogenicity of 7 -valent pneumococcal conjugate vaccine versus 23 -valent polysaccharide vaccine in adults aged 50-80 years. Clin Infect Dis. 2009;49(9): 1318-1325.

45. Grabenstein JD, Manoff SB. Pneumococcal vaccines in adults: assessing the evolving evidence. Vaccine. 2011;29(37):6149-6154.

46. Lazarus R, Clutterbuck E, Yu LM, et al. A randomized study comparing combined pneumococcal conjugate and polysaccharide vaccination schedules in adults. Clin Infect Dis. 2011;52(6):736-742.

47. Dransfield MT, Nahm MH, Han MK, et al. Superior immune response to protein-conjugate versus free pneumococcal polysaccharide vaccine in chronic obstructive pulmonary disease. Am J Respir Crit Care Med. 2009;180(6):499-505.

48. Dransfield MT, Harnden S, Burton RL, et al. Long-term comparative immunogenicity of protein conjugate and free polysaccharide pneumococcal vaccines in chronic obstructive pulmonary disease. Clin Infect Dis. 2012;55(5):e35-e44.

49. Baxendale HE, Johnson M, Keating SM, et al. Circulating pneumococcal specific plasma and memory B cells in the elderly two years after pneumococcal conjugate versus polysaccharide vaccination. Vaccine 2010;28(42):6915-6922.

50. Baxendale HE, Keating SM, Johnson M, Southern J, Miller E, Goldblatt D. The early kinetics of circulating pneumococcal-specific memory B cells following pneumococcal conjugate and plain polysaccharide vaccines in the elderly. Vaccine. 2010;28(30):4763-4770.

51. Grabenstein JD, Manoff SB. Pneumococcal polysaccharide 23-valent vaccine: long-term persistence of circulating antibody and immunogenicity and safety after revaccination in adults. Vaccine. 2012;30(30):4435-4444.

52. Cheng A, Chang SY, Tsai MS, et al. Long-term immune responses and comparative effectiveness of one or two doses of 7-valent pneumococcal conjugate vaccine (PCV7) in HIV-positive adults in the era of combination antiretroviral therapy. J Int AIDS Soc. 2016;19(1):20631.

53. Ben-Shimol S, Givon-Lavi N, Leibovitz E, Raiz S, Greenberg D, Dagan R. Near-elimination of otitis media caused by 13-valent pneumococcal conjugate vaccine (PCV) serotypes in southern Israel shortly after sequential introduction of 7 -valent/13-valent PCV. Clin Infect Dis. 2014;59(12):1724-1732.

54. Greenberg D, Givon-Lavi N, Ben-Shimol S, Ziv JB, Dagan R. Impact of PCV7/PCV13 introduction on community-acquired alveolar pneumonia in children $<5$ years. Vaccine. 2015;33(36):4623-4629.

55. Griffin MR, Mitchel E, Moore MR, Whitney CG, Grijalva CG. Declines in pneumonia hospitalizations of children aged $<2$ years associated with the use of pneumococcal conjugate vaccines: Tennessee, 1998-2012. MMWR Morb Mortal Wkly Rep. 2014;63(44):995-998.
56. Lau WC, Murray M, El-Turki A, et al. Impact of pneumococcal conjugate vaccines on childhood otitis media in the United Kingdom. Vaccine. 2015;33(39):5072-5079.

57. Marom T, Tan A, Wilkinson GS, Pierson KS, Freeman JL, Chonmaitree T. Trends in otitis media-related health care use in the United States, 2001-2011. JAMA Pediatr. 2014;168(1):68-75.

58. Loo JD, Conklin L, Fleming-Dutra KE, et al. Systematic review of the indirect effect of pneumococcal conjugate vaccine dosing schedules on pneumococcal disease and colonization. Pediatr Infect Dis J. 2014; 33(Suppl 2):S161-S171

59. van Hoek AJ, Sheppard CL, Andrews NJ, et al. Pneumococcal carriage in children and adults two years after introduction of the thirteen valent pneumococcal conjugate vaccine in England. Vaccine. 2014;32(34):4349-4355.

60. Schmoele-Thoma B, Jackson LA, Greenberg RN, et al. Immunogenicity of 13-valent pneumococcal conjugate vaccine in immunocompetent older adults with stable underlying medical conditions. $J$ Vaccines Immun. 2015;3(2):7-12.

61. van Werkhoven CH, Huijts SM, Bolkenbaas M, Grobbee DE, Bonten MJ. The impact of age on the efficacy of 13-valent pneumococcal conjugate vaccine in elderly. Clin Infect Dis. 2015;61(12):1835-1838.

62. Falkenhorst G, Remschmidt C, Harder T, Hummers-Pradier E, Wichmann O, Bogdan C. Effectiveness of the 23-valent pneumococcal polysaccharide vaccine (PPV23) against pneumococcal disease in the elderly: systematic review and meta-analysis. PLoS One. 2017; 12(1): 0169368.

63. Kraicer-Melamed H, O'Donnell S, Quach C. The effectiveness of pneumococcal polysaccharide vaccine 23 (PPV23) in the general population of 50 years of age and older: a systematic review and meta-analysis. Vaccine. 2016;34(13):1540-1550.

64. Suzuki M, Dhoubhadel BG, Ishifuji T, et al. Serotype-specific effectiveness of 23-valent pneumococcal polysaccharide vaccine against pneumococcal pneumonia in adults aged 65 years or older: a multicentre, prospective, test-negative design study. Lancet Infect Dis. 2017;17(3):313-321.

65. Centers for Disease Control and Prevention. Use of 13-valent pneumococcal conjugate vaccine and 23-valent pneumococcal polysaccharide vaccine for adults with immunocompromising conditions: recommendations of the Advisory Committee on Immunization Practices (ACIP). MMWR Morb Mortal Wkly Rep. 2012;61(40):816-819.

66. Kobayashi M, Bennett NM, Gierke R, et al. Intervals between PCV13 and PPSV23 vaccines: recommendations of the Advisory Committee on Immunization Practices (ACIP). MMWR Morb Mortal Wkly Rep. 2015;64(34):944-947.

67. Esposito S, Bonanni P, Maggi S, et al. Recommended immunization schedules for adults: clinical practice guidelines by the ESCMID Vaccine Study Group (EVASG), European Geriatric Medicine Society (EUGMS) and the World Association for Infectious Diseases and Immunological Disorders (WAIDID). Hum Vaccin Immunother. 2016;12(7):1777-1794.

68. Greenberg RN, Gurtman A, Frenck RW, et al. Sequential administration of 13-valent pneumococcal conjugate vaccine and 23-valent pneumococcal polysaccharide vaccine in pneumococcal vaccine-naive adults 60-64 years of age. Vaccine. 2014;32(20):2364-2374

69. Schwarz TF, Flamaing J, Rumke HC, et al. A randomized, double-blind trial to evaluate immunogenicity and safety of 13 -valent pneumococcal conjugate vaccine given concomitantly with trivalent influenza vaccine in adults aged $\geq 65$ years. Vaccine. 2011;29(32):5195-5202.

70. Frenck RW Jr, Gurtman A, Rubino J, et al. Randomized, controlled trial of a 13-valent pneumococcal conjugate vaccine administered concomitantly with an influenza vaccine in healthy adults. Clin Vaccine Immunol. 2012;19(8):1296-1303.

71. Woodhead M, Blasi F, Ewig S, et al. Guidelines for the management of adult lower respiratory tract infections: full version. Clin Microbiol Infect. 2011;17(Suppl 6):E1-E59.

72. Global Initiative for Chronic Obstructive Lung Disease. Global Strategy for the Diagnosis, Management and Prevention of Chronic Obstructive Pulmonary Disease. Bethesda, MD: GOLD; 2017. 
73. Murphy TF, Sethi S. Bacterial infection in chronic obstructive pulmonary disease. Am Rev Respir Dis. 1992;146(4):1067-1083.

74. Wilson R. A vicious circle hypothesis operating during infective exacerbations of chronic bronchitis. Monaldi Arch Chest Dis. 1994;49(2): 159-164.

75. Alfageme I, Vazquez R, Reyes N, et al. Clinical efficacy of antipneumococcal vaccination in patients with COPD. Thorax. 2006;61(3): 189-195.

76. Furumoto A, Ohkusa Y, Chen M, et al. Additive effect of pneumococcal vaccine and influenza vaccine on acute exacerbation in patients with chronic lung disease. Vaccine. 2008;26(33):4284-4289.

77. Poole PJ, Chacko E, Wood-Baker RW, Cates CJ. Influenza vaccine for patients with chronic obstructive pulmonary disease. Cochrane Database Syst Rev. 2006;(1):CD002733.

78. Christenson B, Pauksen K, Sylvan SP. Effect of influenza and pneumococcal vaccines in elderly persons in years of low influenza activity. Virol J. 2008;5:52.

79. Gilchrist SA, Nanni A, Levine O. Benefits and effectiveness of administering pneumococcal polysaccharide vaccine with seasonal influenza vaccine: an approach for policymakers. Am J Public Health. 2012;102(4):596-605.

80. Nichol KL. The additive benefits of influenza and pneumococcal vaccinations during influenza seasons among elderly persons with chronic lung disease. Vaccine. 1999;17(Suppl 1):S91-S93.

81. Laxminarayan R, Matsoso P, Pant S, et al. Access to effective antimicrobials: a worldwide challenge. Lancet. 2016;387(10014):168-175.

82. Braeter U, Schulz M, Goffrier B, Schulz M, Ihle P, Bätzing-Feigenbaum $\mathrm{J}$. [Pneumococcal vaccine in GKV-insured persons in the age range of 60 to 64 years: regionalized analysis on the implementation of the recommendations of the Standing Committee on Vaccination Commission based on national contract medical billing data]. 2016. German. Available from: https://www.versorgungsatlas.de/themen/alle-analysennach-datum-sortiert/?tab=6\&uid=74. Accessed October 11, 2017.

83. Carreno-Ibanez LV, Esteban-Vasallo MD, Dominguez-Berjon MF, et al. Coverage of and factors associated with pneumococcal vaccination in chronic obstructive pulmonary disease. Int J Tuberc Lung Dis. 2015;19(6):735-741.

84. Giese C, Mereckiene J, Danis K, O’Donnell J, O’Flanagan D, Cotter S. Low vaccination coverage for seasonal influenza and pneumococcal disease among adults at-risk and health care workers in Ireland, 2013: the key role of GPs in recommending vaccination. Vaccine. 2016;34(32): 3657-3662.

85. Guthmann JP. [National vaccination coverage survey, France, January 2011. Influenza vaccination coverage in target groups and measure of vaccine effectiveness: diphtheria-tetanus-poliomyelitis (dTP) and antipneumococcal vaccination coverage in persons aged 65 years old or above]. 2011. French. Available from: http:// invs.santepubliquefrance.fr/Publications-et-outils/Rapports-etsyntheses/Maladies-infectieuses/2011/Enquete-nationale-de-couverture-vaccinale-France-janvier-2011. Accessed October 1, 2017.

86. Steens A, Vestrheim DF, de Blasio BF. Pneumococcal vaccination in older adults in the era of childhood vaccination: public health insights from a Norwegian statistical prediction study. Epidemics. 2015;11: 24-31.
87. Public Health England. Pneumococcal polysaccharide vaccine (PPV) coverage report, England, April 2014 to March 2015. 2015. Available from: https://www.gov.uk/government/uploads/system/uploads/ attachment_data/file/448406/hpr2615_ppv.pdf. Accessed October 1, 2017.

88. Ipsos. PneuVUE: a new view into pneumonia among older adults. 2016. Available from: https://www.ipsos-mori.com/researchpublications/ publications/1816/PneuVUE-A-New-View-into-Pneumonia-AmongOlder-Adults.aspx. Accessed January 12, 2017.

89. Blasi F, Aliberti S, Bonanni P, Mantero M, Odone A, Signorelli C. [Pneumococcal vaccination in adults: recommendations from the Italian Society of Respiratory Medicine (SIMeR) and the Italian Society of Hygiene, Preventive Medicine and Public Health (SItI)]. Epidemiol Prev. 2014;38(6 Suppl 2):147-151. Italian.

90. Portugal Directorate of Disease Prevention and Health Promotion Services. [Vaccination against Streptococcus pneumoniae infections in groups at increased risk for invasive pneumococcal disease (IPD): adults ( $\geq 18$ years of age).] 2015. Portuguese. Available from: https://www.dgs.pt/directrizes-da-dgs/normas-e-circulares-normativas/ norma-n-0112015-de-23062015.aspx. Accessed October 11, 2017.

91. Gonzalez-Romo F, Picazo JJ, Garcia Rojas A, et al. [Consensus document on pneumococcal vaccination in adults at risk by age and underlying clinical conditions: 2017 update]. Rev Esp Quimioter. 2017; 30(2):142-168. Spanish.

92. France Ministry of Social Affairs and Health. [Calendar of vaccinations and vaccine recommendations 2017]. 2017. French. Available from: http://social-sante.gouv.fr/vaccins-vaccination. Accessed October 1, 2017.

93. Joint Committee on Vaccination and Immunisation. Minutes of the meeting on 4 February 2015. 2015. Available from: https://app.box. com/s/iddfb4ppwkmtjusir2tc/1/2199012147/27417264008/1. Accessed October 1, 2017.

94. Standing Committee on Vaccination (STIKO). Recommendations of the Standing Committee on Vaccination (STIKO) at the Robert Koch Institute - effective: August 2016. 2016. Available from: http://www. rki.de/EN/Content/infections/Vaccination/recommandations/34_2016_ engl.pdf. Accessed October 1, 2017.

95. Kim YK, LaFon D, Nahm MH. Indirect effects of pneumococcal conjugate vaccines in national immunization programs for children on adult pneumococcal disease. Infect Chemother. 2016;48(4):257-266.

96. Weycker D, Bornheimer R, Shea KM, Sato R, Pelton SI. Excess exacerbations following pneumonia in adults with pre-existing heart failure (HF) or chronic obstructive pulmonary disease (COPD). Poster presented at: 10th International Symposium on Pneumococci and Pneumococcal Diseases (ISPPD); June 26-30, 2016; Glasgow, Scotland.

97. Suaya JA, Jiang Q, Bonten M, et al. Post-hoc analysis of the 13-valent polysaccharide conjugate vaccine efficacy against vaccine-serotype pneumococcal community acquired pneumonia in at-risk older adults. Poster 634, presented at: the 10th International Symposium on Pneumococci and Pneumococcal Diseases (ISPPD); June 29, 2016; Glasgow. Scotland.
International Journal of COPD

\section{Publish your work in this journal}

The International Journal of COPD is an international, peer-reviewed journal of therapeutics and pharmacology focusing on concise rapid reporting of clinical studies and reviews in COPD. Special focus is given to the pathophysiological processes underlying the disease, intervention programs, patient focused education, and self management protocols.

\section{Dovepress}

This journal is indexed on PubMed Central, MedLine and CAS. The manuscript management system is completely online and includes a very quick and fair peer-review system, which is all easy to use. Visit $\mathrm{http}: / / \mathrm{www}$.dovepress.com/testimonials.php to read real quotes from published authors. 